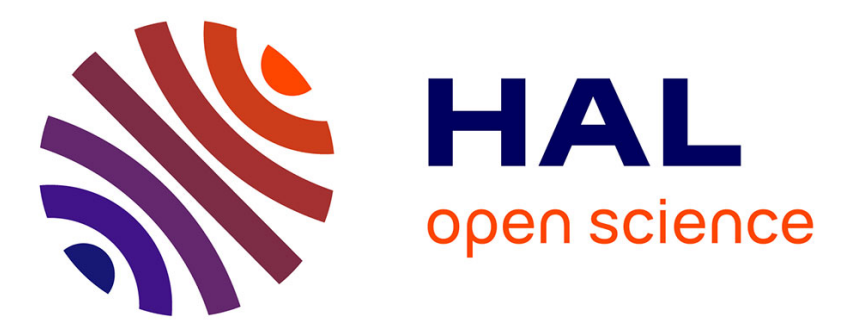

\title{
Tracking Growing Axons by Particle Filtering in 3D+t Fluorescent Two-Photon Microscopy Images
}

Huei-Fang Yang, Xavier Descombes, Charles Kervrann, Caroline Medioni, Florence Besse

\section{- To cite this version:}

Huei-Fang Yang, Xavier Descombes, Charles Kervrann, Caroline Medioni, Florence Besse. Tracking Growing Axons by Particle Filtering in 3D+t Fluorescent Two-Photon Microscopy Images. ACCV Asian Conference on Computer Vision, Nov 2012, Daejeon, South Korea. hal-00740966

\section{HAL Id: hal-00740966 https://hal.inria.fr/hal-00740966}

Submitted on 11 Nov 2012

HAL is a multi-disciplinary open access archive for the deposit and dissemination of scientific research documents, whether they are published or not. The documents may come from teaching and research institutions in France or abroad, or from public or private research centers.
L'archive ouverte pluridisciplinaire HAL, est destinée au dépôt et à la diffusion de documents scientifiques de niveau recherche, publiés ou non, émanant des établissements d'enseignement et de recherche français ou étrangers, des laboratoires publics ou privés. 


\title{
Tracking Growing Axons by Particle Filtering in $3 \mathrm{D}+t$ Fluorescent Two-Photon Microscopy Images
}

\author{
Huei-Fang Yang ${ }^{1}$, Xavier Descombes ${ }^{1}$, Charles Kervrann ${ }^{2}$, Caroline Medioni ${ }^{1}$, \\ and Florence Besse ${ }^{1}$ \\ 1 MORPHEME, INRIA/I3S/IBV, 06903 Sophia Antipolis Cedex, France \\ 2 SERPICO, INRIA Rennes, 35042 Rennes Cedex, France
}

\begin{abstract}
Analyzing the behavior of axons in the developing nervous systems is essential for biologists to understand the biological mechanisms underlying how growing axons reach their target cells. The analysis of the motion patterns of growing axons requires detecting axonal tips and tracking their trajectories within complex and large data sets. When performed manually, the tracking task is arduous and time-consuming. To this end, we propose a tracking method, based on the particle filtering technique, to follow the traces of axonal tips that appear as small bright spots in the $3 \mathrm{D}+t$ fluorescent two-photon microscopy images exhibiting low signal-to-noise ratios (SNR) and complex background. The proposed tracking method uses multiple dynamic models in the proposal distribution to predict the positions of the growing axons. Furthermore, it incorporates object appearance, motion characteristics of the growing axons, and filament information in the computation of the observation model. The integration of these three sources prevents the tracker from being distracted by other objects that have appearances similar to the tracked objects, resulting in improved accuracy of recovered trajectories. The experimental results obtained from the microscopy images show that the proposed method can successfully estimate trajectories of growing axons, demonstrating its effectiveness even under the presence of noise and complex background.
\end{abstract}

\section{Introduction}

Analyzing how growing axons correctly reach their target neurons is essential for biologists to better understand the development of a nervous system. The advances in fluorescence imaging technology, such as two-photon microscopy [1], have generated amounts of imaging data that enable the analysis of the dynamic behavior of developing neurons in real time and in three dimensions (3D). Analysis of the properties of axon growth requires detecting axonal tips that can appear as small bright spots and tracing their trajectories in low signal-tonoise ratios (SNR) image sequences. Figure 1 shows some examples of part of maximum intensity projection (MIP) images of the fluorescent two-photon microscopy volumes. As can be seen, in addition to high level of noise, the obtained 
volumes contain some bright spots that have appearances similar to the axonal tips and other biological structures that increase the difficulty in tracing the axonal tips. These factors make it difficult to manually identify the growing axonal tips and follow their trajectories. Besides, manual tracing by human experts to obtain the trajectories of axons is tedious and time-consuming. Therefore, developing automated tracking methods that produce reliable trajectories for further analysis is highly required.

Numerous methods have been proposed in the literature for tracking biological spot-like particles. A great majority of the tracking methods are based on two-step approach: the object detection/localization step and the data association step [2-4]. The detection step aims at locating the objects of interest (e.g. the bright spots) by utilizing intensity thresholding [5], wavelet transform [6], or Gaussian fitting methods [7]. Since the detection step has been performed, the data association step solves the object correspondence problem between consecutive detection images [5]. Generally, these two steps are performed independently. Therefore, because the detection results may contain missing or incorrectly detected objects, the data association step tends to produce wrongly associated trajectories.

Recently, Bayesian tracking approaches have received much attention and have been successfully applied to spot tracking [8-10]. One advantage of these approaches is that they integrate the detection and data association steps in a unified framework, which produces more accurate trajectories. Moreover, they can deal with nonlinear and non-Gaussian tracking problems. The Bayesian tracking is usually implemented by using a sequential Monte Carlo (SMC) method, that is the so-called particle filtering (PF) [11]. Particle filtering techniques use prior knowledge of the dynamics of the objects to predict the object state modes and use all the available information (noisy measurements) to compute optimally the posterior probability density function (pdf).

In this paper, we propose a tracking method, based on the particle filtering technique, to follow the trajectories of the individual growing axons in the fluorescent two-photon microscopy image sequences. The proposed method incorporates the important characteristics of growing axons. First, multiple dynamic models are used to better predict the locations of axonal tips. Furthermore, the proposed method incorporates information related to object appearance, motion characteristics of the growing axons, and filaments in the computation of the likelihood function. The integration of these three sources prevents the tracker from being distracted by other objects that have appearances similar to the tracked objects. It ensures that the tracker can successfully follow the traces of the individual axonal tips, resulting in obtaining more accurate trajectories.

The remainder of this paper is organized as follows. Section 2 presents an overview of the particle filtering framework, and Section 3 elaborates on the proposed particle filtering based tracking method that incorporates the characteristics of growing axons. The experimental results obtained from synthetic data and a $3 \mathrm{D}+t$ fluorescent two-photon microscopy data set are shown in Section 4 , followed by conclusions and future work in Section 5 . 


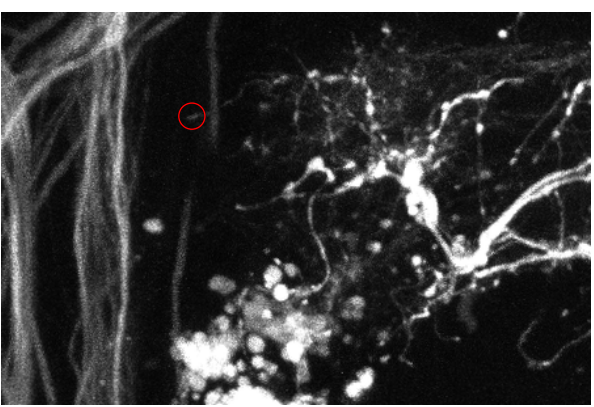

(a) MIP at time 86

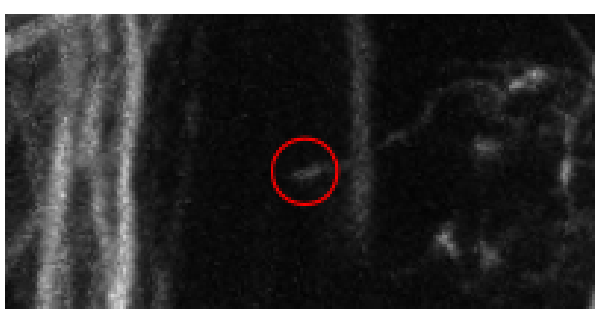

(c) close-up of (a)

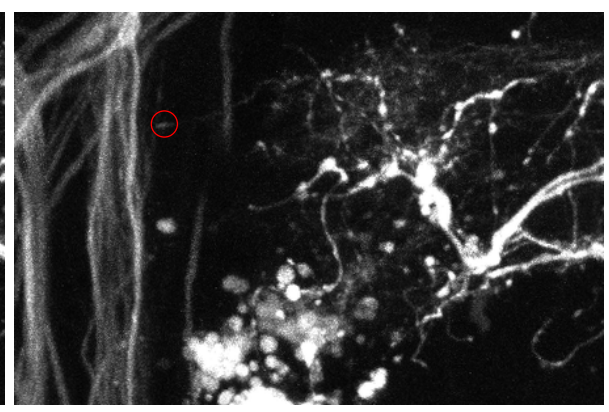

(b) MIP at time 90

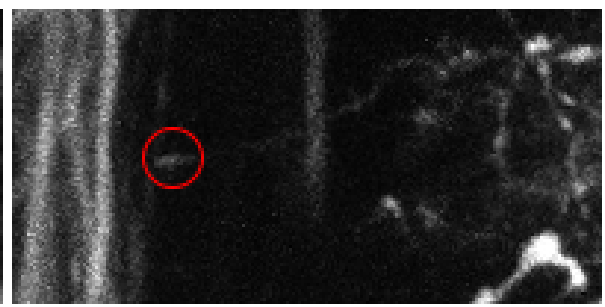

(d) close-up of (b)

Fig. 1. Examples of part of maximum intensity projection (MIP) of the fluorescent two-photon microscopy volumes. (a) and (b) show part of MIPs from different times and demonstrate the dynamics of the growing axons. (c) and (d) are the close-ups of (a) and (b), respectively. The axonal tips are visualized as small bright spots, indicated by red circles. The 3D microscopy volumes are of low SNR ratios and contain other biological structures and some bright spots that have appearances similar to the axonal tips. These factors increase the difficulty in identifying and tracking the axonal tips.

\section{$2 \quad$ Particle Filtering}

The Bayesian tracking aims at recursively estimating the posterior probability density function, $p\left(\mathrm{~s}_{t} \mid \mathrm{z}_{1: t}\right)$, which describes the state $\mathrm{s}_{t}$ at time $t$ given a series of (noisy) measurements $\mathrm{z}_{1: t}$ from time 1 to time $t$. The estimation consists of two steps: prediction and updating.

Prediction The prediction step relies on the Chapman-Kolmogorov equation, that is,

$$
p\left(\mathrm{~s}_{t} \mid \mathrm{z}_{1: t-1}\right)=\int p\left(\mathrm{~s}_{t} \mid \mathrm{s}_{t-1}\right) p\left(\mathrm{~s}_{t-1} \mid \mathrm{z}_{t-1}\right) d \mathrm{~s}_{t-1},
$$

where $p\left(\mathrm{~s}_{t} \mid \mathrm{s}_{t-1}\right)$ decides the prior dynamics.

Updating The update step uses the Bayes' rule to modify the prior density and obtain the posterior density when a measurement $\mathrm{z}_{t}$ is available:

$$
p\left(\mathrm{~s}_{t} \mid \mathrm{z}_{1: t}\right) \propto p\left(\mathrm{z}_{t} \mid \mathrm{s}_{t}\right) p\left(\mathrm{~s}_{t} \mid \mathrm{z}_{1: t-1}\right)
$$


where $p\left(\mathrm{z}_{t} \mid \mathrm{s}_{t}\right)$ represents the likelihood function (observation model).

This recursive estimation of the posterior pdf is intractable, and the particle filtering is used as a numerical approximation. The particle filtering [12] uses a set of $N_{s}$ samples, each of which associates with a weight, $\left\{\mathrm{s}_{t}^{i}, w_{t}^{i}\right\}_{i=1}^{N_{s}}$, to represent the posterior $p\left(\mathrm{~s}_{t} \mid \mathrm{z}_{1: t}\right)$ :

$$
p\left(\mathrm{~s}_{t} \mid \mathrm{z}_{1: t}\right) \approx \sum_{i=1}^{N_{s}} w_{t}^{i} \delta\left(\mathrm{s}_{t}-\mathrm{s}_{t}^{i}\right)
$$

where $\delta(\cdot)$ is the Dirac delta function, and $\sum_{i}^{N_{s}} w_{t}^{i}=1$. These samples are propagated through time to approximate the posterior pdf at the subsequent steps via a proposal distribution $q\left(\mathrm{~s}_{t} \mid \mathrm{s}_{t-1}, \mathrm{z}_{t}\right)$. The importance weight of each sample, $w_{t}^{i}$, is updated as follows:

$$
w_{t}^{i} \propto w_{t-1}^{i} \frac{p\left(\mathrm{z}_{t} \mid \mathrm{s}_{t}^{i}\right) p\left(\mathrm{~s}_{t}^{i} \mid \mathrm{s}_{t-1}^{i}\right)}{q\left(\mathrm{~s}_{t}^{i} \mid \mathrm{s}_{t-1}^{i}, \mathrm{z}_{t}\right)} .
$$

The final state at time $t$ can be computed as the maximum a posteriori (MAP) estimator:

$$
\hat{\mathrm{s}}_{t}=\underset{\mathrm{s}_{t}^{i}}{\operatorname{argmax}} p\left(\mathrm{~s}_{t}^{i} \mid \mathrm{z}_{t}\right) \forall i=1 \ldots N_{s} .
$$

When applied to the tracking problems after a few iterations, the particle filtering has the degeneracy problem that only few particles carry significant weights. A resampling procedure is then required to generate a new set of equally weighted samples based on the importance weights.

\section{Proposed Method}

The section details the proposed method by starting with the description of the multiple dynamic models used in the proposal distribution. Then we present the observation model that integrates information related to object appearance, object motion, and filaments.

\subsection{Multiple Dynamic Models}

Before describing the dynamic models, the state of an axonal tip is first introduced. The state of an axonal tip at time $t$ is represented by s $\mathrm{s}_{t}=\left(x_{t} y_{t} z_{t} \dot{x_{t}} \dot{y_{t}} \dot{z}_{t}\right)^{T}$, where $x_{t}, y_{t}$, and $z_{t}$ denote the position of the tip in the Cartesian coordinate and $\dot{x}_{t}, \dot{y}_{t}$, and $\dot{z}_{t}$ represent the velocities in the $x, y$, and $z$ directions. To better predict the position of the axonal tip at time $t$, the proposal distribution contains two dynamic models, defined as

$$
q\left(\mathrm{~s}_{t} \mid \mathrm{s}_{t-1}, \mathrm{z}_{t}\right)=\pi_{1} p\left(\mathrm{~s}_{t} \mid \mathrm{s}_{t-1}\right)+\pi_{2} p\left(\mathrm{~s}_{t} \mid \mathrm{s}_{t-1}, \mathrm{~s}_{t-2}\right),
$$


where $p\left(\mathrm{~s}_{t} \mid \mathrm{s}_{t-1}\right)=\mathcal{N}\left(\mathrm{s}_{t} \mid \mathrm{s}_{t-1}, \sigma_{1}^{2}\right)$ and $p\left(\mathrm{~s}_{t} \mid \mathrm{s}_{t-1}, \mathrm{~s}_{t-2}\right)=\mathcal{N}\left(\mathrm{s}_{t} \mid \mathrm{s}_{t-1}+v_{t-1}, \sigma_{2}^{2}\right)$ are the first-order Markov transition and the second-order Markov transition, respectively, where $v_{t-1}$ is the velocity at time $t-1$, given by $v_{t-1}=\left(\dot{x}_{t-1} \dot{y}_{t-1} \dot{z}_{t-1}\right)$. The notation $\mathcal{N}\left(:, \sigma^{2}\right)$ represents a Gaussian with variance $\sigma^{2}$. The first-order Markov transition models the phenomenon that the growing axons sometimes stop growing for a short period of time and then resume the growth process, and the second-order transition captures the typical motion behavior of growing axons, which tends to follow the growing direction at time $t-1$. The weights $\pi_{1}$ and $\pi_{2} \triangleq 1-\pi_{1}$ control the mixture for each dynamic model.

\subsection{Observation Model}

The proposed observation model combines information about object appearance, object motion, and filaments in order to better follow the axonal tips.

Appearance Likelihood The appearance likelihood aims at modeling the prior knowledge about the image formation process in the microscopy imaging systems (point spread function (PSF)). Now, let $I_{t}^{\text {ideal }}(x, y, z)$ be the ideal intensity, without the effect of noise, at point $(x, y, z)$ at time $t$, and is given by [13]

$$
I_{t}^{i d e a l}(x, y, z)=\sum_{i} P_{i, t}(x, y, z)+b_{t},
$$

where $P_{i, t}$ is the object profile, and $b_{t}$ is the background intensity. Here, Gaussian functions are used to approximate the PSFs of the microscopy.

However, because of the noise generated during the image acquisition process, the observed intensity $I_{t}^{o b s}$ is the addition of the ideal intensity $I_{t}^{\text {ideal }}$ and the noise $N_{t}$ that is assumed to be additive and Gaussian:

$$
I_{t}^{o b s}=I_{t}^{i d e a l}+N_{t}
$$

To compute the likelihood, the residual image between the observed image and the ideal image is first obtained by

$$
R_{t}=I_{t}^{o b s}-I_{t}^{i d e a l}
$$

After the residual image is obtained, the appearance likelihood is therefore computed as

$$
p\left(R_{t} \mid \mathrm{s}_{t}\right)=\frac{1}{Z_{1}} \exp \left(-\alpha_{1}|| R_{t} \|_{2}\right),
$$

where $\alpha_{1}$ is a constant, and $Z_{1}$ is a normalizing constant.

Motion Model It is established in biology that axons reach their target cells in the developing nervous system by the guidance of molecular gradients $[14,15]$. Accordingly, the growing axons do not abruptly change their growing directions. In other words, the turning angles of the growing axons between consecutive time 
sequences are small, leading to smooth trajectories. The likelihood is designed to model this characteristic of the movement patterns of growing axons. Assume that $r_{t}$ is the motion direction vector at time $t$. The angle difference, $d_{t}$, between the motion direction vectors at time $t$ and at time $t-1$ is given as

$$
D_{t}=\arccos \left(r_{t}, r_{t-1}\right) .
$$

Therefore the motion likelihood is defined as

$$
p\left(D_{t} \mid \mathrm{s}_{t}\right)=\frac{1}{Z_{2}} \exp \left(-\alpha_{2} D_{t}\right),
$$

where $\alpha_{2}$ is a constant, and $Z_{2}$ is a normalizing constant.

Filament Model Frangi's vessel enhancement filter [16] is applied to enhance the filaments in the images. The basic idea of Frangi's method is to use the eigenvalues of the Hessian (second-order information) to measure the vesselness. Now, let $\lambda_{k}$ represent the $k$-th smallest eigenvalue obtained from a Hessian matrix (i.e., $\left.\left|\lambda_{1}\right| \leq\left|\lambda_{2}\right| \leq\left|\lambda_{3}\right|\right)$, and the eigenvalues of an ideal tubular structure in a 3 D volume has the following properties: (1) $\left|\lambda_{1}\right| \approx 0,(2)\left|\lambda_{1}\right| \ll\left|\lambda_{2}\right|$, and (3) $\lambda_{2} \approx \lambda_{3}$.

To quantitatively distinguish the tubular-like structure from blob-like and plate-like structures, two geometric ratios $R_{A}$ and $R_{B}$ are defined. They are $R_{A}=\frac{\left|\lambda_{2}\right|}{\left|\lambda_{3}\right|}$ and $R_{B}=\frac{\left|\lambda_{1}\right|}{\sqrt{\left|\lambda_{2} \lambda_{3}\right|}}$, where $R_{A}$ is for distinguishing between platelike and line-like structures, and $R_{B}$ accounts for the deviation from a bloblike structure. Besides, because background pixels have small magnitudes of the eigenvalues, a measurement $S$ is used to distinguish the tubular-like structures from background and is defined as $S=\sqrt{\sum_{j} \lambda_{j}^{2}}$.

Finally, a vesselness function $V_{\delta}(p)$ that integrates the above 3 measurements, $R_{A}, R_{B}$, and $S$, at scale $\delta$ is given by

$V_{\delta}(p)= \begin{cases}0 & \text { if } \lambda_{2}>0 \text { or } \lambda_{3}<0 \\ \left(1-\exp \left(-\frac{R_{A}(p)^{2}}{2 \gamma^{2}}\right)\right) \exp \left(-\frac{R_{B}(p)^{2}}{2 \beta^{2}}\right)\left(1-\exp \left(-\frac{S(p)^{2}}{2 c^{2}}\right)\right) & \end{cases}$

where $\gamma, \beta$, and $c$ are parameters that control the sensitivity of the line filter to the measures $R_{A}, R_{B}$, and $S$, respectively, and $p$ denotes a voxel.

Equation (13) is analyzed at different scales to measure the vesselness in an image so the final estimate of the vesselness is the maximum filter response among all the scales, that is

$$
V(p)=\max _{\delta_{\min } \leq \delta \leq \delta_{\max }} V_{\delta}(p),
$$

where $\delta_{\min }$ and $\delta_{\max }$ are the minimum and maximum scales at which relevant structures are expected to be found. Figure 2(b) shows the MIP image by applying Frangi's vessel enhancement filter. The tubular-like structures are enhanced 


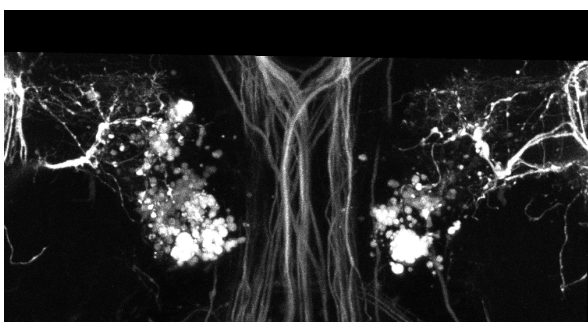

(a) MIP of the original volume

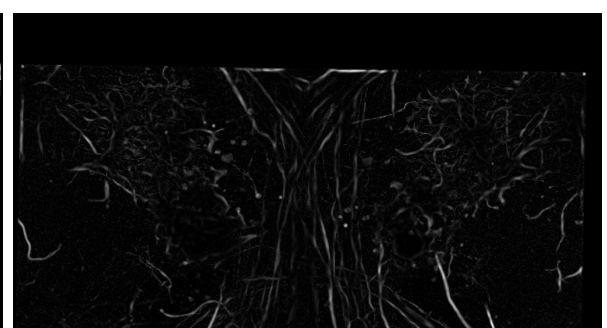

(b) MIP of applying Frangi's filter to (a)

Fig. 2. MIPs of the original volume and of the Frangi's filtered volume. The tubular-like structures are enhanced and the other structures including background are suppressed in (b) after Frangi's filter is applied to (a). Note that the filter is applied to 3D volumes, and the MIPs of these 3D volumes are shown here for the visualization purpose.

and the others are suppressed when compared to the original MIP shown in Figure 2(a).

Let $l$ be the trajectory (a line segment) that an axon moves from time $t-1$ to time $t$ and $\overline{V_{l, t}}$ be the average value of the measured vesselness through this trajectory. The filament likelihood is therefore given by

$$
p\left(\overline{V_{l, t}} \mid \mathrm{s}_{t}\right)=\frac{1}{Z_{3}} \exp \left(-\alpha_{3}\left(1-\overline{V_{l, t}}\right)\right),
$$

where $\alpha_{3}$ is a constant, and $Z_{3}$ is normalizing constant.

Joint Likelihood Assuming the independence of object appearance, object motion, and filament measurement, the joint likelihood for the observation model is defined as

$$
p\left(\mathrm{z}_{t} \mid \mathrm{s}_{t}\right)=p\left(R_{t} \mid \mathrm{s}_{t}\right) p\left(D_{t} \mid \mathrm{s}_{t}\right) p\left(\overline{V_{l, t}} \mid \mathrm{s}_{t}\right) .
$$

\section{Experimental Results}

The experiments were carried out on synthetic data and a fluorescent microscopy data set to evaluate the performance of the proposed method. The performance is quantitatively measured by comparing the computer generated tracking results to the ground truth based on evaluation metrics.

\subsection{Experiments on Synthetic Data}

Data To validate our method, a synthetic data set, with a size of $100 \times 100 \times 35$, that simulates the movement of growing axons is generated. Different levels of Gaussian noise with standard deviation (STD) of 5 and 7 were added to the synthetic data, resulting in a total of 2 image sequences. Figures 3(a) and 3(b) show the generated synthetic images corrupted by Gaussian noise with STD $=5$ and $\mathrm{STD}=7$, respectively. 


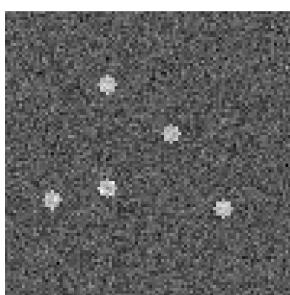

(a) $\operatorname{STD}=5$

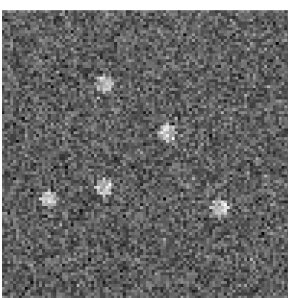

(b) $\mathrm{STD}=7$

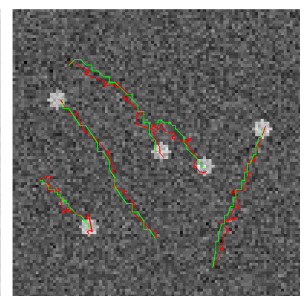

(c) $\mathrm{STD}=5$

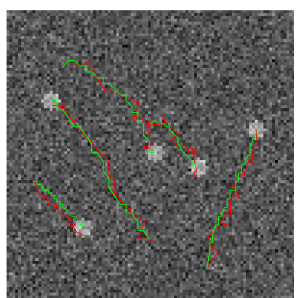

(d) $\mathrm{STD}=7$

Fig. 3. Generated synthetic images with different levels of Gaussian noise and tracking results. (a) and (b) show the synthetic images by adding Gaussian noise with STD $=5$ and STD $=7$, respectively. (c) and (d) show the tracking results in red and the ground truth in green. The results produced by the proposed method are consistent with the ground truth. Note that the contrast of images is enhanced for visualization.

Results To quantitatively measure the performance of the proposed tracking method, the tracked trajectories were compared to manually generated ground truth by measuring the root mean square error (RMSE) [10] of positions between the tracked trajectories and the ground truth. The RMSE is defined as

$$
\mathrm{RMSE}=\sqrt{\frac{1}{K} \sum_{k=1}^{K} \mathrm{MSE}_{k}^{2}}
$$

where $K$ is the number of independent runs, and

$$
\mathrm{MSE}_{k}^{2}=\frac{1}{M} \sum_{m=1}^{M} \frac{1}{T_{m}} \sum_{t \in T_{m}}\left\|r_{m, t}-\hat{r}_{m, t}^{k}\right\|^{2}
$$

where $r_{m, t}$ is the true position of object $m$ at time $t, \hat{r}_{m, t}^{k}$ is the estimated position, and $T_{m}$ is the number of frames where object $m$ exists.

Figures 3(c) and 3(d) show the comparison between the tracking results generated by the proposed method, shown in red, and the ground truth, shown in green. By visual comparison, the computer generated tracks are consistent with the ground truth.

Using RMSE as the performance measure metric, the localization errors for the synthetic data set corrupted by Gaussian noise with STD $=5$ and STD $=7$ were 2.5565 and 2.6174 pixels, respectively, which indicates that the proposed method can correctly follow the movement of growing axons.

\subsection{Experiments on Fluorescent Two-Photon Microscopy Data}

Data $\mathrm{A} 3 \mathrm{D}+t$ fluorescent two-photon microscopy data set of the intact Drosophila fly brains was used to evaluate the performance of the proposed tracking method. The volume size at each time is $1012 \times 548 \times 16(x \times y \times z)$ voxels, and 170 such 


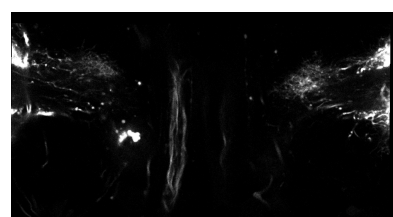

(a) Original image

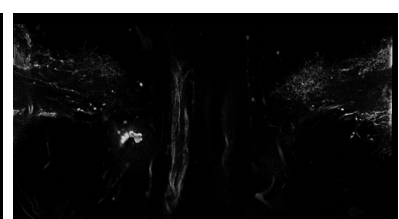

(b) Objects

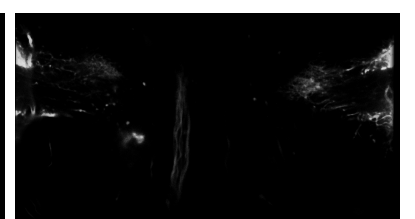

(c) Background

Fig. 4. Obtained moving objects and partial background by using the HullkGround software. As can be seen, the original frame (a) contains noise and complex background, and the background subtraction step decomposes the original image into an image containing moving objects (b) and an image containing slightly moving background (c).

volumes are generated. Each volume has a size of $133.75 \times 72.36 \times 12 \mu \mathrm{m}^{3}$, with a resolution of $0.132 \times 0.132 \times 0.8 \mu \mathrm{m}^{3} /$ voxel. To analyze the dynamics of axons in the developing nervous system, the trajectories of the individual growing axons have to be tracked.

Preprocessing The fluorescent microscopy data set was obtained by imaging the living tissue at different time points so the subsequent volumes had to be aligned to obtain the correct motion patterns of growing axons. In addition, the raw images contain noise and complex background that have to be removed in order to obtain more accurate trajectories. Hence, prior to the tracking, two preprocessing steps, registration and background subtraction, were performed.

Registration Motion2D [17], a software to estimate 2D parametric motion models in an image sequence, was used to register the images in order to eliminate the living sample's drift problem caused during the image acquisition process. To register the $3 \mathrm{D}+t$ image sequence, the MIP image at each time was first obtained, and the registration process was performed to estimate an affine motion model for each pair of consecutive MIP images. The obtained motion models were then used to register each individual frame. Though a 3D registration method that registers consecutive volumes may be more appropriate for this case, we observed that this $2 \mathrm{D}$ registration approach was able to give satisfying results.

Background Subtraction As demonstrated in Figure 1, the obtained images are noisy and contain other biological structures (background) that may affect the tracking performance. To remove noise and partial background, the HullkGround software [18], which performs background subtraction by convex hull estimation, was used to decompose the registered data set into two dynamic components: (1) an image sequence containing moving objects and (2) an image sequence showing the slightly moving background. Figures 4(b) and 4(c) show the image containing moving objects and the image containing the background, respectively. As can be seen, because of the high complexity of the background, only partial background is removed from the original image shown in Figure 4(a). As 


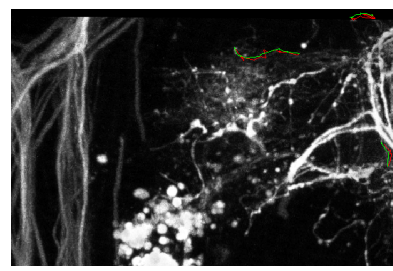

(a)

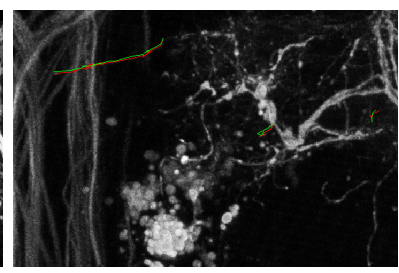

(b)

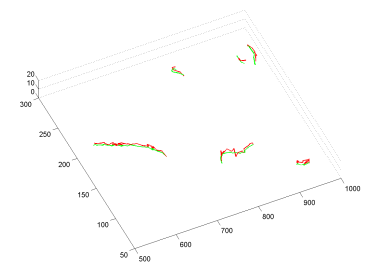

(c)

Fig. 5. Visual comparison between the tracking results of the proposed method and the manually created ground truth in $2 \mathrm{D}$ and $3 \mathrm{D}$. The red trajectories are produced by the proposed method, and the green are the ground truth manually created by the expert; both are overlaid on the MIPs (a) and (b) and visualized in 3D (c). The computer generated tracks are consistent with the ground truth in general, with minor differences between the estimated positions and the ground truth positions. The differences are caused by the noise and by the effect of complex background.

a result, the image shown in Figure 4(b) still contains some parts of the background. Even so, the proposed tracking method still yielded better results on the moving objects images than the original images.

Tracking Results The proposed tracking method was applied to the tracking of growing axons in the image sequence containing moving objects, which was obtained by background subtraction. The initial positions of the axonal tips were manually set by the user, and the tracking algorithm followed the traces of the growing axons in the subsequent images. In the experiments, a set of 200 samples was used in the particle filter. The weights (i.e. $\pi_{1}$ and $\pi_{2}$ in Equation (6)) for the two dynamic models were set to 0.5 . The parameters $\alpha_{1}, \alpha_{2}$, and $\alpha_{3}$ in the observation model were set to $0.2,2.5$, and 0.25 , respectively. Figure 5 shows the $2 \mathrm{D}$ and $3 \mathrm{D}$ visual comparisons between the tracked trajectories produced by the tracking algorithm, shown in red, and the ground truth tracks, shown in green. As can be seen, the computer generated trajectories are consistent with the manually created ground truth in general, with minor differences between the estimated positions and the true positions, which is caused by the noise and complex background. This indicates that the proposed method was able to successfully follow the traces of axonal tips.

Using RMSE as the performance measure metric, for all the tracks, the overall localization error of the proposed method is 4.56 pixels.

The mean computation time using a Matlab implementation of the proposed method on a laptop with Intel Core i5 CPU $(2.53 \mathrm{GHz})$ and 4GB memory was 1.8926 seconds for tracking one axonal tip at a time step. Note that this computation time does not include the time required for reading frames and applying Frangi's filter. 
Performance Comparison Between Different Likelihood Combinations To investigate how each likelihood term in the observation model affects the tracking results, we evaluated the tracking performance with 3 different likelihood combinations: (1) appearance and motion likelihoods and (2) appearance and filament likelihoods, and (3) appearance, motion, and filament likelihoods. The appearance likelihood is commonly used in most of the tracking methods and hence is retained in each combination. Hence, the main focus is on the investigation of how motion and filament likelihoods affect the performance. The localization errors for the 3 combinations in order were $7.51,7.87$, and 4.83 pixels. The integration of appearance, motion, and filament likelihoods in the observation model increases the localization accuracy.

\section{Conclusions and Future Work}

This paper presented a tracking method, based on the particle filtering technique, to follow the trajectories of the individual growing axons in fluorescent microscopy images. The proposed method uses multiple dynamic models in the proposal distribution in order to predict the positions of the axonal tips. Besides, information related to object appearance, motion, and filament is integrated in the computation of the likelihood. The fused information prevents the tracker from being distracted by the other objects that have appearances similar to the axonal tips; consequently, the proposed tracking method can successfully estimate the trajectories of the axonal tips in the image sequences that are noisy and have complex background. The experimental results obtained from a $3 \mathrm{D}+t$ fluorescent two-photon microscopy image sequence demonstrate the effectiveness of the proposed method.

Future work includes designing a detection method to automatically initialize the tracking process and quantitatively analyzing the tracked trajectories to gather statistical parameters of the growing axons. Current detection methods usually produce many false positives in detecting the axonal tips and thus a detection algorithm that is suitable for task is required. For analysis of the axons, the parameters, such as curvatures, turning angles, and growing lengths, will be extracted and used for mathematically modeling the growing patterns of axons. We expect that this statistical analysis and modeling will provide valuable insight into a better understanding of the characteristics of growing axons in the developing nervous system.

Acknowledgement. This work was partially supported by ARC DATA (INRIA).

\section{References}

1. Helmchen, F., Denk, W.: Deep tissue two-photon microscopy. Natural Methods 2 (2005) 932-940 
2. Genovesio, A., Liedl, T., Emiliani, V., Parak, W.J., Coppey-Moisan, M., OlivoMarin, J.C.: Multiple particle tracking in 3-D+t microscopy: method and application to the tracking of endocytosed quantum dots. IEEE Trans. Image Processing 15 (2006) 1062-1070

3. Matov, A., Applegate, K., Kumar, P., Thoma, C., Krek, W., Danuser, G., Wittmann, T.: Analysis of microtubule dynamic instability using a plus-end growth marker. Nature methods 7 (2010) 761-768

4. Sbalzarini, I.F., Koumoutsakos, P.: Feature point tracking and trajectory analysis for video imaging in cell biology. J. Structural Biology 151 (2005) $182-195$

5. Apgar, J., Tseng, Y., Fedorov, E., Herwig, M.B., Almo, S.C., Wirtz, D.: Multipleparticle tracking measurements of heterogeneities in solutions of actin filaments and actin bundles. Biophysical J. 79 (2000) 1095-1106

6. Olivo-Marin, J.C.: Extraction of spots in biological images using multiscale products. Pattern Recognition 35 (2002) 1989-1996

7. Zhang, B., Zerubia, J., Olivo-Marin, J.C.: Gaussian approximations of fluorescence microscope point-spread function models. Applied Optics 46 (2007) 1819-1829

8. Cardinale, J., Rauch, A., Barral, Y., Székely, G., Sbalzarini, I.F.: Bayesian image analysis with on-line confidence estimates and its application to microtubule tracking. In: Proc. IEEE Int'l Symp. Biomedical Imaging. (2009) 1091-1094

9. Smal, I., Meijering, E.H.W., Draegestein, K., Galjart, N., Grigoriev, I., Akhmanova, A., van Royen, M.E., Houtsmuller, A.B., Niessen, W.J.: Multiple object tracking in molecular bioimaging by rao-blackwellized marginal particle filtering. Medical Image Analysis 12 (2008) 764-777

10. Smal, I., Draegestein, K., Galjart, N., Niessen, W.J., Meijering, E.H.W.: Particle filtering for multiple object tracking in dynamic fluorescence microscopy images: Application to microtubule growth analysis. IEEE Trans. Med. Imaging 27 (2008) 789-804

11. Isard, M., Blake, A.: CONDENSATION - conditional density propagation for visual tracking. Int'l J. Computer Vision 29 (1998) 5-28

12. Arulampalam, M.S., Maskell, S., Gordon, N.J., Clapp, T.: A tutorial on particle filters for online nonlinear/non-gaussian Bayesian tracking. IEEE Trans. Signal Processing 50 (2002) 174-188

13. Chenouard, N., de Chaumont, F., Bloch, I., Olivo-Marin, J.C.: Improving 3D tracking in microscopy by joint estimation of kinetic and image models. In: Medical Image Computing and Computer-Assisted Intervention, MIAAB workshop. (2008)

14. Goodhill, G.J., Gu, M., Urbach, J.S.: Predicting axonal response to molecular gradients with a computational model of filopodial dynamic. Neural Computation 16 (2004) 2221-2243

15. Krottje, J.K., van Ooyen, A.: A mathematical framework for modeling axon guidance. Bull Math Biol 69 (2007) 3-31

16. Frangi, A.F., Niessen, W.J., Vincken, K.L., Viergever, M.A.: Multiscale vessel enhancement filtering. In: Medical Image Computing and Computer-Assisted Intervention. (1998) 130-137

17. Odobez, J.M., Bouthemy, P.: Robust multiresolution estimation of parametric motion models. J. Vis. Comm. and Image Representation 6 (1995) 348-365

18. Chessel, A., Cinquin, B., Bardin, S., Salamero, J., Kervrann, C.: Computational geometry-based scale-space and modal image decomposition: application to light video-microscopy imaging. In: Conf. Scale Space and Variational Methods. (2009) $770-781$ 